\title{
SDR application for implementing an integrated UMTS/WiMAX PHY-layer architecture
}

\author{
Olga Zlydareva \\ Department of Information and \\ Communication Technology (DIT) \\ University of Trento \\ Via Sommarive 14, \\ POVO 38050 TN, Italy \\ +393298479684 \\ olga.zlydareva@dit.unitn.it
}

\author{
Claudio Sacchi \\ Department of Information and \\ Communication Technology (DIT) \\ University of Trento \\ Via Sommarive 14, \\ POVO $38050 \mathrm{TN}$, Italy \\ +390461883907 \\ sacchi@dit.unitn.it
}

\begin{abstract}
Emerging standards in the area of mobile services, such as Worldwide Interoperability for Microwave Access (WiMAX) and Universal Mobile Telecommunication System (UMTS), require new capabilities from mobile devices. As a result, these devices should know how to download and correctly install reconfigurable modules. For this purpose, the Software Defined Radio (SDR) technology is used at present. SDR allows co-existence of different independent standards, protocols, and services. This signal processing approach is widespread due to the great importance of reprogramming and reconfiguring of mobile devices. Because of the availability of SDR technique in device architecture, a user can update and replace necessary services without changing hardware. In this paper, we present a new solution of SDR implementation for UMTS/WiMAX system. Furthermore, we consider common blocks in our system in detail and demonstrate the implementation of the framework model in object-oriented style using SystemC.
\end{abstract}

\section{Keywords}

Software Defined Radio, UMTS, WiMAX, Physical layer, SystemC.

\section{INTRODUCTION}

Current research works in wireless communication technologies focus on providing variety of services and maintaining high bit rate [1]. The possibility to be reachable in any place and anytime has also become much demanded. That is why companies try to find possible solutions to satisfy these requirements. To this end, there is a need to devise a structure which will be able to support retuning mobile terminals according to signal reception [2]. Existing approaches assume that a user should purchase a detached device for each standard, because most of them have their own specification of frequencies range, types of modulation, coding scheme, and access to the environment. Therefore, a mobile operator has to provide support for every wireless system separately. To solve this problem, the Software Defined Radio (SDR) technology comes in handy [3].

SDR is one possible way to realize the structure of the device with high mobility, flexibility, and reconfigurability [4]. This

Permission to make digital or hard copies of all or part of this work for personal or classroom use is granted without fee provided that copies are not made or distributed for profit or commercial advantage and that copies bear this notice and the full citation on the first page. To copy otherwise, to republish, to post on servers or to redistribute to lists, requires prior specific permission and/or a fee. MOBIMEDIA 2007, August 27-29, Nafpaktos, Greece

Copyright $\odot 2007$ ICST 978-963-06-2670-5

DOI 10.4108/ICST.MOBIMEDIA2007.1728 technology can provide seamless shifting between existing airinterface standards. The basic definition of SDR can be sketched as follows: SDR is a system which can realize connection between different hardware units, arranged in a radio node, and govern the whole process by using software functions. This kind of control widens perspectives of SDR systems' development as it is the main part of mobile devices. Moreover, the chance to handle the system consisting of different radio-functional units by applying software commands can be relevant for the reconfiguration and reprogramming [5].

Many 2G standards such as Interim Standard 54 (IS-54), and IS136, IS-95, Global System Mobile Communication (GSM) and Personal Digital Cellular (PDC) are widely used in the mobile communication area. At the same time, 3G standards such as: International Mobile Telecommunication-2000 (IMT-2000), Code Division Multiple Access (CDMA) 2000 and Universal Mobile telecommunication System (UMTS) [6] are widespread. Although mobile communication systems have improved their data transmission parameters, that are bit rate reaches up to 2 Mbps and the real-video format can be transmitted, wireless systems such as various specifications of IEEE 802.11, Bluetooth and emerging specifications of IEEE 802.16 have become popular [7].

Present technologies oblige their users to buy a particular device for each type of communication standard, and operators have to prepare base stations for each system. However, current needs require to seamlessly handling user's equipment from one cellular standard to another or from the cellular network to hotspot. For this case, emerging multi-standards, multi-bands, multi-modes [8], and multi-protocols systems have been developed. The capability to integrate as many as possible diverse services in a single terminal is extended. Therefore, SDR technique can serve as the fundamental technology. SDR allows system reconfiguration and reprogramming by using only software commands. These commands help to build required architecture of the transceiver. Thus, SDR is the mechanism supporting system rebuild on request. The work of SDR includes hardware adaptability by software means that is by downloading the necessary software from the network and installation. All these steps require no skills and no efforts from the user.

The usage of new standards, such as WiMAX (IEEE 802.16e2005) [9] for providing a fast Internet connection with ability to transmit large data files, and UMTS in telephony family, are spreading everywhere [10]. Thereby, examination of the 
possibility to realize at least two standards over one common element base which will be handled and rebuilt by SDR [11] has become relevant.

In our work, we propose a novel approach to handle the signal processing for two standards - WiMAX and UMTS. The structural level of our development includes the baseband (BB) level of the transceiver architecture. We present the SDR module framework which will be able to arrange the digital processing module. This work will be realized in the high-level programming language as SystemC. The general structure will be constructed by a number of modules for each particular component of UMTS and WiMAX signal processing chain, and also by the modules for common units. This work aims to demonstrate how this system works. We show that the system can recognize the type of the signal and select a particular mechanism for processing according to the modulation type, code scheme and frequency range.

In this paper, we consider the structure of WiMAX signal processing by particular blocks corresponding to the baseband processing. From the point of view of UMTS signal, we assume that the physical layer (PHY) of a signal processing is described by bit sequence blocks up to RF linkage. These blocks from each subsystem can be adapted by SDR technique in our proposed UMTS/WiMAX system. For this purpose, we defined each component of the BB level processing and tried to find the parts which can be common blocks for our whole SDR system. For UMTS, we selected the UMTS FDD (Frequency Division Duplex) [12] mode and for WiMAX, we picked the IEEE 802.162004 standard with OFDM physical layer (FFT with 256 points) and FDD mode as well [13].

The UL baseband architectures of WCDMA (Wide-band Code Division Multiplexing) and OFDM (Orthogonal Frequency Division Multiplexing) have different performance, but share some similar parts. Thus, we present the description of these parts and a possible solution to realize them as common blocks using SDR.

The rest of the paper is structured as follows. Section 2 describes the proposed realization of the SDR technique with WiMAX/UMTS system. Section 3 sketches the description of possible common blocks. Then, Section 4 presents preliminary results obtained to the moment by software simulations. Finally, the conclusions and directions of the future work are drawn in Section 5.

\section{SDR FOR UMTS/WIMAX SYSTEM}

There are different classes of services in telecommunications. Some works for providing transmission of voice and short message services; others can handle high data rate transmission of real-time video and vast range of Internet services. The emerging standards of personal communication require new capabilities from user equipment (UE). UE must satisfy different types of modulation, code schemes, and access to environment. Moreover, UE has to retune frequency band, because in many cases different standards work on its own passband both for uplink signal and for downlink signal. Thus, there is no overlap of frequency bands. Our choice of the air-interface is based on WiMAX and UMTS standards, because each of them orients to providing the different classes of services. Together they can give an optimal decision for information transmission.
WiMAX is the modern broadband wireless connection for Metropolitan Area Network (MAN), standardized by Institute of Electrical and Electronics Engineers as IEEE 802.16 with specifications [14]. Accordingly, the network architecture requires less time to be built. The networks realized on WiMAX base represent more flexible, simple, and scalable structures. This new technology is aimed to:

- provide wideband access in cases where DSL connection cannot be applied;

- $\quad$ solve the Last mile problem;

- $\quad$ enable simultaneous connection for a large number of users;

- transmit audio-, video- and multimedia files with a high bit rate, up to 75 Mbps;

- $\quad$ provide access anywhere and anytime;

- guarantee non-line-of-sight capability;

- $\quad$ provide broadband wireless access for huge quantity of users for short time period (for example internet access during the conference in hotel).

The specification 802.16-2004 is a perspective direction for development of urban and rural area networks, especially in cases where the signal has a lot of distortions and interferences during its propagation. One of the most important advantages is the support of non-line-of-sight that occurs due to reducing the frequency band below $11 \mathrm{GHz}$ and using the Orthogonal Frequency Division Multiplexing (OFDM) or Orthogonal Frequency Division Multiplexing Access (OFDMA). This type of signal processing structure for baseband level is determined as the multi-carrier transmission technique and perfectly suitable for uplink and downlink transmissions. The main motivation for using the OFDM technique is transmission of multiple carriers in parallel. The problem of interference can be resolved by using Fast Fourier Transform (FFT) [15].

Thereby, general parameters of physical layer are critical for our work. We have to be precise in describing all possible variations of the bit sequence transformation for each UL block. The block diagram of the baseband level for WiMAX UL is depicted in Figure 1.

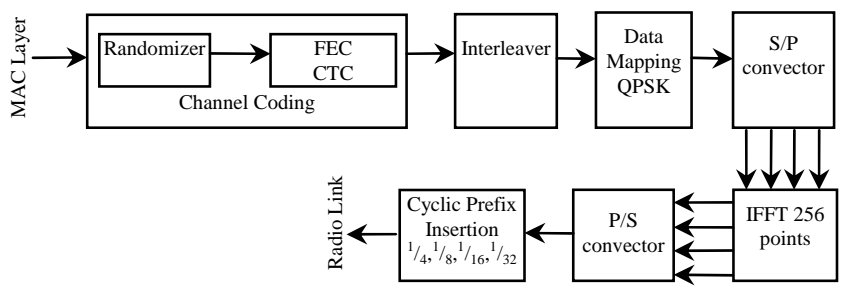

Figure 1: The block scheme of WiMAX PHY layer architecture in UL direction.

UMTS is one of the third generation cellular technologies, which can support real-time video, and also number of other services such as voice call, video/audio broadcasting, mobile TV, web browsing, mobile office, and services based on user location, maps and guidebooks, GPS, mobile e-commerce and payment of tickets. The chain of the UMTS signal processing is presented in Figure 2.

The multi-user finding is achieved by implementation WCDMA as a UMTS PHY layer. The possibility of working with variable number of users leads to Inter-Symbol and Intra-Symbol 
Interferences. Reducing channel intercrossing occurs at the expense of direct sequence spread spectrum mechanism, which is implemented over $5 \mathrm{MHz}$ band per carrier in UMTS. Due to the signal spreading by pseudo-noise sequence, each user is separated from another one by his or her own unique code. Moreover, the high bit rate (for example $960 \mathrm{kbps}$ ) for uplink connection can be achieved by the combination of the changeable spreading gain technique and the fixed chip rate (3.84 Mcps). The high downlink rate can be obtained by using QPSK and turbo coding. WCDMA technology is also intended for multiple simultaneous accesses on the same frequency band. With the appearance of the next generation wireless connection, such as WiMAX, which supports huge data transmission, and advances in phone capabilities, the co-existence both of them in the same device has become the vital direction in telecommunication area.

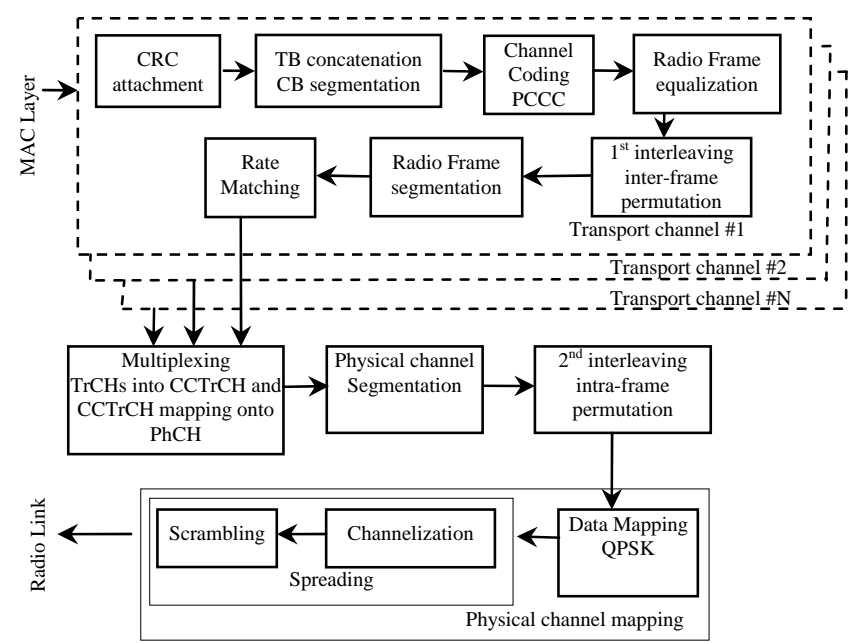

Figure 2: The block scheme of UMTS PHY layer architecture in UL direction.

The SDR technique is the most suitable for the realization of seamless switching between different types of access into environment, because it allows flexible system retuning. The SDR method is known as the way of platform reconfiguration and reprogramming, because of its controlling and handling software functions. This technology allows running different software modules which are located on generic hardware platform, for example DSP or FPGA. This hardware platform provides the radio function performances, arranged by integrated SDR module and can be implemented as the storage of components corresponding to software functions.

In order to achieve better performances for WiMAX as well as for UMTS, the new design of the baseband physical layer for mobile devices based on commanding by the SDR control module is presented in this paper. The block scheme of the signal processing is given in Figure 3.

Among different schemes of the signal processing for WiMAX and UMTS at the baseband level [16], we selected the SDR technology to be applied for reconfiguring and reprogramming the signal processing (SP) block. The special chains of processed blocks are built by the SDR modules which are included in the WiMAX/UMTS SDR library. Then, the SDR library is loaded into DSP or FPGA. Main principles of the work of the SDR modules and the SDR library are schematically illustrated in Figure 4.

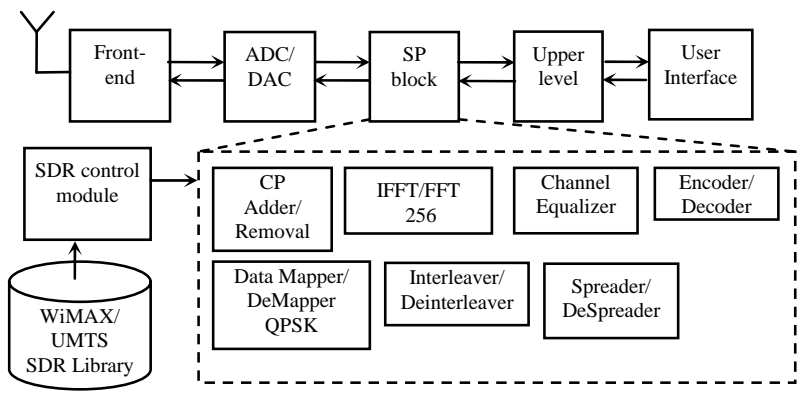

Figure 3: The main block scheme for the transceiver based on SDR modules in the baseband level.

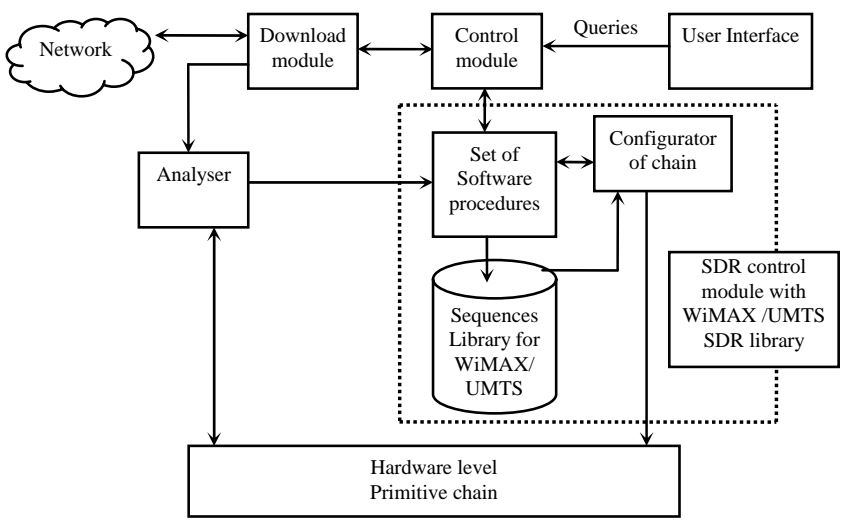

Figure 4: The block scheme of detailed work of proposed SDR module and SDR library.

On a user's query, the Control module sends a simple request containing the application parameters to the Network, and then receives the information about the design settings, which is necessary to download the needed services. After that, the Analyzer sends the required parameters to the Set of Software procedures that communicates with the Sequences Library. This library stores the commands describing main and particular functions of signal processing. Then, a set of these functions is sent to the Configurator of chain. The Configurator builds an appropriate order of the executed processing and communicates to the hardware level, which provides the element base. Finally, the user terminal starts to work according to the established parameters.

\section{MAIN BLOCKS DETERMINATION}

In this section, we describe the base structure of the physical layers of both UMTS and WiMAX which are based on WCDMA technique and OFDM technique respectively. After that, we separate out the main blocks which can be implemented as SDR blocks.

In OFDM baseband level architecture shown in Figure 1, there are the following blocks: Channel Coding, Interleaver, Constellation Mapper (Data Mapping process), Serial-to-Parallel (S/P) 
Converter, IFFT with 256 points, Parallel-to-Serial (P/S) Converter, and Cyclic Prefix Adder.

Figure 2 shows the WCDMA architecture. It contains such blocks as: CRC attachment, Transport Block (TB) concatenation and Code Block (CB) segmentation, Channel Coding, Radio frame (Rf) equalization, $1^{\text {st }}$ interleaving, Rf segmentation, Rate Matching, Multiplexing, Physical channel segmentation, $2^{\text {nd }}$ interleaving, Data Mapping, and Spreading (Channelization and Scrambling).

In each baseband architecture, the common blocks and particular blocks are present. We can organize them in the following order: Channel Coding scheme, Interleaver, and Data Mapping.

\subsection{Channel coding}

In this subsection, we first explain the UMTS encoder structure and then the WiMAX encoder. Each of them has it own parameters. In the proposed model, we combine encoder structures in one SDR block using a digital procedure description. Since in OFDM the turbo coding scheme is existed as an optional performance, we can define the turbo coding block as a common block in our UMTS/WiMAX architecture.

One of the key parts of baseband level signal processing is Channel Coding. There are two possible methods of Channel Coding in UMTS physical layer architecture:

- convolutional coding (CC);

- turbo coding (TC).

In UMTS specification [17], the choice of coding scheme and coding rate depends on the type of channel, see Table 1.

Table 1. The correspondence TrCH types to Coding scheme and Coding rate

\begin{tabular}{|c|c|c|}
\hline TrCh type & Coding scheme & Coding rate \\
\hline $\begin{array}{c}\text { BCH, PCH, } \\
\text { RACH }\end{array}$ & \multirow{2}{*}{ Convolutional coding } & $1 / 2$ \\
\cline { 3 - 3 } & & $1 / 2,1 / 3$ \\
\cline { 3 - 3 } DCH, FACH & Turbo coding & $1 / 3$ \\
\hline
\end{tabular}

In our framework model, we work with dedicated channels (DCH) of both transport and physical layer. Consequently, the turbo coding scheme can be chosen with a coding rate equal to 1/3. The Turbo encoder component in the UMTS framework consists of two constituent encoders and an internal interleaver. The encoder block scheme is provided in Figure 5.

This scheme is based on a Parallel Concatenated Convolutional Code (PCCC) with two 8-statets constituent encoders and one internal interleaver. Each encoder is a binary Recursive Systematic Convolutional (RSC) code, which is the key component of Turbo Coding. The RSC principle works as Linear Feedback Shift-Register and behaves as pseudo-random scrambler. The prime value of shift registers shall be equal to zero when input bits are being encoded. The tail bits are padded in terms of trellis termination of turbo code processing. The next important block of the Turbo encoder is the Turbo code internal interleaver which forms input bits to a rectangular matrix (RM) with padding, intra-row and inter-row permutation of RM and output bits sequence from the RM with pruning.

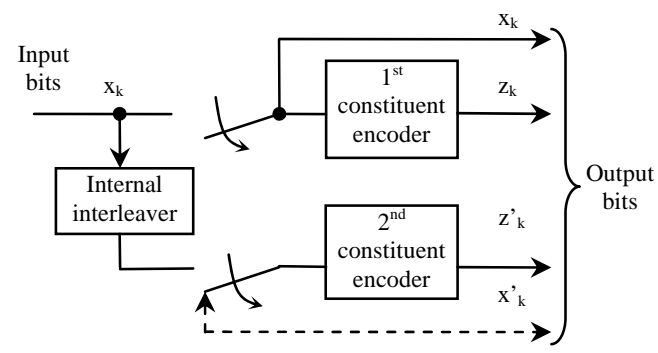

Figure 5. Block scheme of UMTS Turbo encoder.

The code block size used in bits input varies between 40 and 5114. Three types of RM exist for different block sizes: 5, 10 and 20 rows. The number of columns depends on the input bit sequence and prime number of inter-row permutation. After all permutations the bit sequence are written into the $2^{\text {nd }}$ constituent encoder by taking columns by columns out. There is a code block in the output of the Turbo encoder consisting of three tails bits from $1^{\text {st }}$ constituent encoder, second constituent encoder and input bits to the Turbo encoder.

One of the Turbo coder parameters is the transfer function, which characterizes relation between input and output. For the 8-states constituent code of PCCC the transfer function is defined as follows:

$$
G(D)=\left[1, \frac{g_{1}(D)}{g_{0}(D)}\right],
$$

where $g_{1}(D)$ and $g_{0}(D)$ are polynomial functions for feedback branch and parity bit respectively. These polynomials are defined as:

$$
\begin{aligned}
& g_{0}(D)=1+D^{2}+D^{3} \\
& g_{1}(D)=1+D+D^{3} .
\end{aligned}
$$

The next part described is the Coding Scheme in WiMAX baseband level. There are three different possibilities to realize the coding process in Forward Error Correction subblock. One possible solution is to use Reed-Solomon outer code and ratecompatible convolutional inner code. Another way is to use Block Turbo Coding. Finally, the last possible approach is to adopt Convolutional Turbo Coding (CTC) scheme. Since Turbo Coding scheme is more robust and advanced scheme, we consider it as a perspective direction for emerging standard IEEE 802.16-2004. In our model, we took the Convolutional Turbo Coding in order to apply it like SDR block for both cases. Moreover, this specification is included in the WCDMA coding scheme.

The constituent encoder is one of the central parts of the CTC encoder that uses a double binary Circular RSC code. The block scheme of encoder is shown in Figure 6. 


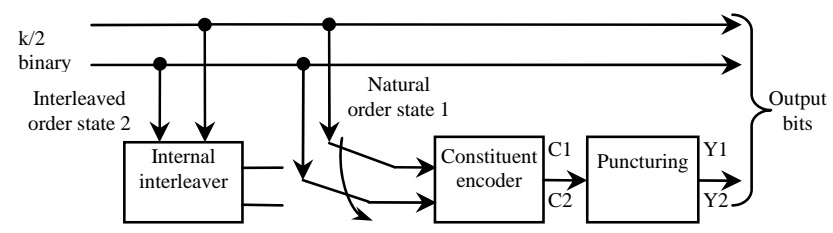

Figure 6. Convolutional Turbo Code encoder block scheme for WiMAX baseband processing.

The polynomials distinguishing the connections inside the Constituent encoder are defined in symbol notation as follows:

Y parity bit $\quad 1+D^{2}+D^{3}$

Feedback branch $1+D+D^{3}$

However, in more precise description there are some discrepancies in each of Turbo Coding implementations. The comparison between two schemes is realized in Table 2. As it is shown in the table below, the main difference between coding schemes is that double bit sequence is present at input of encoder in OFDM process chain. In addition, inside the RSC, the second bit should allow for future bit sequence transformation.

Table 2. Turbo Code Comparison for WCDMA and OFDM implementations

\begin{tabular}{|c|c|c|}
\hline Characteristics & WCDMA & OFDM \\
\hline Type & Binary, 8-state & Duo-Binary, 8-state \\
\hline Termination & Tail bits & Circular \\
\hline Polynomials & $13,15,17$ & 13,15 \\
\hline Rates & $1 / 4,1 / 3,1 / 2$ & $1 / 2$ up to $7 / 8$ \\
\hline
\end{tabular}

\subsection{Interleaving}

The interleaving process allows avoiding the burst error during signal transmission. In both cases, two permutations are performed. From the UMTS point of view there is first interleaving made on a $\mathrm{TrCH}$ bit sequence. The inter-columns permutation is done by the matrix with determined number of columns. The number of columns depends on the size of Transmission Time Interval (TTI) (see Table 3). The main parameter of permutation scheme choice is TTI which is defined as a set of serially concatenated transport blocks and indicates how often data from the higher layer comes to the physical layer. According to particular permutation pattern the output of $1^{\text {st }}$ interleaving is read as bit sequence.

Table 3. Link between TTI and number of columns

\begin{tabular}{|c|c|c|}
\hline TTI, ms & Number of columns & Permutation patterns \\
\hline 10 & 1 & $<0>$ \\
\hline 20 & 2 & $<0,1>$ \\
\hline 40 & 4 & $<0,2,1,3>$ \\
\hline 80 & 8 & $<0,4,2,6,1,5,3,7>$ \\
\hline
\end{tabular}

The second interleaving is performed in the physical channel. This intra-frame permutation limits the number of columns to 30 , and uses the universal common pattern to minimize the processing load and thus to reduce hardware scale and power consumption. Also here a matrix has a rectangular structure and it is constituted by padding the dummy bits, which are then pruned away in the output of the matrix after the inter-column permutation.

In OFDM, we work with frequency interleaving mechanism, which is aimed to spread the burst errors out in the bit-stream after FEC. This process is accomplished in two step permutation: first sub-carrier permutation and second bit-to-symbol mapping permutation [18]. Thus, the mapping is performed by constellation of adjacent code bits into nonadjacent subcarriers. Then these code bits are mapped onto significant components. The block size equals to 384 coded bits per one interleaved block. This number was defined by the functional dependence interleaver block size and modulation type given that in our framework model, we use the QPSK modulation and the default number of subchannels, i.e., 16. The number of code bits $N_{c p c}$ equals to 2 in each sub-carrier. Thus, we have fixed permutation pattern defined by equation:

$$
m_{k}=\left(N_{c b p s} / 12\right) \cdot k_{\bmod 12}+\text { floor }(k / 12)
$$

where $m_{k}$ is coded bit index after first and before second permutation, $N_{c b p s}$ is number of coded bits per allocated subchannels per OFDM symbol, for our model assume 384, $k$ is code bit index in input and equals to $0,1, \ldots, N_{c b p s}-1$.

Second permutation allows constructing the bit-to-symbol mapping by equation:

$$
j_{k}=m_{k}+\left(m_{k}+N_{c b p s}-\text { floor }\left(12 \cdot m_{k} / N_{c b p s}\right)\right)_{\bmod (1)}
$$

Here $\mathrm{j}_{\mathrm{k}}$ is the code bit index after the second permutation.

The issue is creation of common interleaving block. The way of transformation will depend on kind of characteristic bit sequences. These bit sequences should have the size corresponding to OFDM or WCDMA model.

\subsection{Data mapping}

In this subsection, we describe the data mapping of the bit sequence onto I and Q branches, it is called QPSK constellation. In our UMTS/WiMAX system, we consider QPSK as mapping scheme allocated before spreading transformation of WCDMA signal and before FFT mechanism for the OFDM signal. It is necessary to say that QPSK mapping is a transfer of bit sequence consisting of binary 0 and binary 1 into the inphase $(+1)$ and the quadrature $(-1)$ of the real-valued part respectively. Below we define the similarities and differences between the QPSK mapping of WCDMA signal and OFDM bit sequence in respect to SDR realization.

Since our model is developed for both dedicated transport and physical channels, in followings explanations we speaks only about these types of channels in UMTS chain.

Data Mapping is accomplished after mapping Code Composite Transport Channel (CCTrCH), constituted by multiplexing of few Transport Channels (TrCH), onto Dedicated Physical Channel, as depicted on Figure 7. One PhCH can include just one $\mathrm{CCTrCH}$, in other words one $\mathrm{CCTrCH}$ can be spread between different $\mathrm{PhCH}$, but there is no different CCTrCHs in one $\mathrm{PhCH}$. 


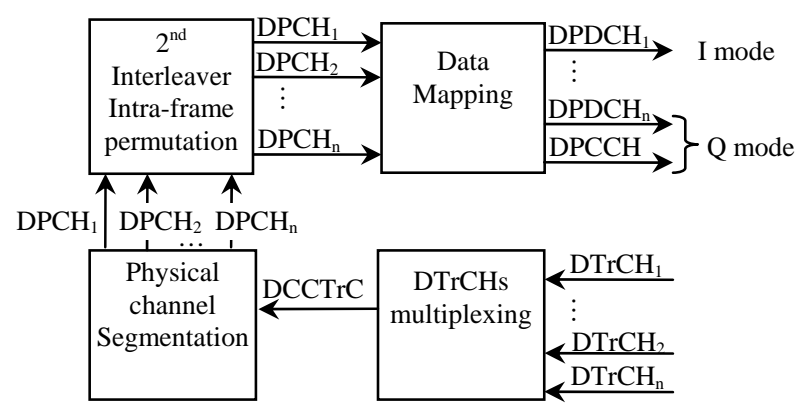

Figure 7. TrCHs multiplexing and mapping onto PhCHs.

In WCDMA UL two types of dedicated physical channels (data and control) are multiplexed with I-Q/code technique which allows the parallel transmission separated different Dedicated Physical Data Channels (DPDCHs) and Dedicated Physical Control Channel (DPCCH) as shown in Figure 8.

First step is the I-Q mapping in this constellation and then applying the combination either channelization code and scrambling or just scrambling code, in case if there is no other PhCHs.

$\left.\begin{array}{|c|c|c|}\hline \mathrm{DPDCH}_{1} & \mathrm{DPDCH}_{2} & \mathrm{DPDCH}_{3} \\ \hline \multicolumn{3}{|c|}{\begin{array}{c}\text { DPCCH } \\ \text { (Control information provided by physical layer) }\end{array}}\end{array}\right\} \begin{gathered}\text { Dedicated } \\ \text { Physical } \\ \text { Channel }\end{gathered}$

\section{Figure 8. Physical layer transmission with parallel} dedicated channels.

Each DPDCH shall be mapped onto I and Q components, and DPCCH can be mapped only onto $\mathrm{Q}$ component. This technique allows the separation of different signals by using the orthogonal phases 0 and $\pi / 2$. The UMTS provides the transmission of up to six DPDCHs and one DPCCH simultaneously.

From WiMAX point of view OFDM signal has a constellation mapper in the signal processing scheme. Different data rates are supported in WiMAX signal processing. It influences onto the mapping technique. This process provides the map of bits into the OFDM symbol description by applying gray-mapped QPSK technique. Here code bits are mapped into I and Q symbol coordinates.

In OFDM case, we perform signal transformation with set of subcarriers separated as data carriers, pilot carriers and null carriers. Data carriers carry information bits. Pilots ensure the synchronization between Transmitter and Receiver parts and channel estimation as well. And nulls are used to avoid InterSymbol Interference, i.e. Guard Interval in time domain, and like the direct current (DC) carrier. There is no any information in nulls carriers. Thus all allocated data subcarriers shall be modulated by Gray-mapped QPSK. In the output of constellation mapper we have I and Q symbols which are organized into OFDM symbol after IFFT block. The general mapping scheme becomes the same for both cases.

This module can be implemented as the common unit for both configurations independently, so that in WCDMA we obtain up to 6 channels and 200 subchannels in OFDM.

\section{SDR SOFTWARE IMPLEMENTATION}

In previous section, we described the common blocks for the proposed architecture. The chain of these blocks can be configured by the SDR control module. Because all our modules have a digital description form and should be written in DSP or FPGA hardware, we can emulate their work in the software application SystemC. This application allows to describe each block separately and to configure the correct chain of required signal processing by calling corresponding functions. The main file links all subfiles into an incorporated system.

The UMTS PHY layer description is not trivial task, because the major part of parameters depends on higher levels. At the beginning we can abstract our model from it and fixed necessary parameters. Since our model consists of different blocks, each of them has its own input and output and posterior code description. The goal is object-oriented model of whole system in software expression.

First we have to describe the block and create the corresponding file. It has been done for each possible model, inasmuch as the UMTS technique supports the adaptive modulation scheme and hence different bit rates, also the WiMAX technique obtains different bit rates by an implementation of adaptive modulation scheme and proper coding. We assume first input parameters as: the developed chain is transmitter side, modulation type is QPSK, since it is available in UMTS and WiMAX transmitter, channel coding scheme is Turbo coding with $1 / 3$ code rate for UMTS and 2/3 code rate for WiMAX, the number of channels depends on input bit sequence.

One of the main objects of the model is the packet file, because it includes procedures of the function description for every type of signal scenarios. The input of framework includes the UMTS input packet and WiMAX input packet, although parameters of packets are determined in the description of packet file.

Next developing step of the proposed structure is the extension and the hierarchical ordering of more complex architecture. This architecture must include descriptions of different information rates and corresponding UE capabilities. Thus the SDR model must be filled by required elements. The particular modules can be defined during this extension. And the hierarchical ordering will store the correct string of called procedures and functions in modules.

This system architecture can be implemented in DSP TMS320 ${ }^{\mathrm{TM}}$ family of Texas Instruments [19]. This DSP family includes set of particular digital processors, which have peculiar to them features. For our purpose following DSPs can be suitable: DaVinci Technology-based Digital Media Processor (TMS320DM644 $\mathrm{x}^{\mathrm{TM}}$ ) and High Performance DSP $\left(\mathrm{TMS} 320 \mathrm{C} 6000^{\mathrm{TM}}\right)$. First one is oriented to multimedia transmission such as: videophones, streaming media and digital TV. Hence it is aimed onto high bit rate transmission with large packet size. Otherwise the second DSP can support video and voice transcoding, packet data processing and acceleration of broadband transmission and imaging applications. The experimental platform (Small Form Factor SDR Evaluation Module) based on DaVinci DSP is existed by LYRTECH [20]. There are key benefits: possibility to apply RF module with required frequency range, separated power control for each processor and availability of all protocol layers implementation 
due to the incorporation between GPP, DSP and FPGA. This platform allows working with WiMAX and cellular equipments.

\section{CONCLUSION AND FUTURE WORK}

In this paper, we considered the position of SDR blocks in common UMTS/WiMAX architecture for mobile terminal. The contact aspects in two different transmission chains in baseband level were presented. We proposed a possible solution to allow the co-existence of different data transmission technologies. Although, there is a problem of UMTS transmission based on single carrier, WiMAX represents a multicarrier transmission thanks to the use of the OFDM technique. The problem can be resolved by the consideration of a direct sequence from a frequency domain. It means that spread spectrum, allowing the avoidance of the inter-channel interference, can be presented by orthogonal frequency components of signal protection.

In sense to develop this point we are going to consider DSSS technology from orthogonal frequency point of view in one of steps in future work. In such a way, it is expected that two different technologies will be approached to each other as closely as possible.

Next steps of the UMTS/WiMAX system development are preparation of the specification and implementation of all possible scenarios. Each scenario will include particular blocks parameters and common description of main blocks. But we have to be carefully in case of main blocks description, because there are a plenty of features.

\section{ACKNOWLEDGMENTS}

The authors would like to thank the company "Arslogica” for the support of this research and especially Luca Debiasi, Fernando Pianegiani and Fabrizio Stefani.

\section{REFERENCES}

[1] Fourty, N., Val, T., Fraisse, P., Mercier, J.-J., "Comparative analysis of new high data rate wireless communication technologies From Wi-Fi to WiMAX”, Proc. of Autonomic and Autonomous Systems and International Conference on Networking and Services, Oct. 2005 pp.66 - 66.

[2] Held, I., Klein, O., Chen, A., Huang, C.-Y., Ma, V., "Receiver Architecture and Performance of WLAN/Cellular Multi-Mode and Multi-Standard Mobile Terminals”, Proc. of IEEE Vehicular Technology Conference 2004, vol. 3, pp. $2248-2253$.

[3] Samukic, A., "UMTS Universal Mobile Telecommunications System: development of standards for the third generation”, Proc. of IEEE Global Telecommunications Conference 1998 (GLOBECOM'98), Nov. 1998, vol.4, pp.1976 - 1983.

[4] Zhigang, L., Wei, L., Yan, Z., Wei, G., “A Multi-standard SDR Base Band Platform”, Proc. of 2003 IEEE International Conference on Computer Networks and Mobile Computing, October 2003, pp. $461-464$.
[5] Fu, P.-W., Chen, K.C., “A Programmable Transceiver Structure of Multi-rate OFDM-CDMA for Wireless Multimedia Communications”, Proc. of IEEE Vehicular Technology Conference, 2001, vol. 3, pp. 1942-1946.

[6] Komara, M., "SDR Architecture Ideally Suited for Evolving 802.16 WiMAX”, published by AirNet Communications, SDR Forum Exhibition, 2004.

[7] Santhi, K.R., Kumaran, G.S., "Migration to 4 G: Mobile IP based Solutions", Proc. of International Conference on Internet and Web Applications and Services/Advanced International Conference, Feb. 2006, pp. 76 - 76.

[8] Xavier, B., Christensen, L., "Cellular/WiFi Multi-Mode Systems”, Proc. of IEEE Radio and Wireless Symposium, Jan. 2006, pp. 3-6.

[9] Wang, F., Ghosh, A., Love, R., Stewart, K., Ratasuk, R., Bachu, R., Sun Y., Zhao Q., "IEEE 802.16e system performance: analysis and simulations". Proc. of IEEE Personal, Indoor and Mobile Radio Communications Symp. 2005, Vol. 2, pp. $900-904$.

[10] Nguyen-Vuong, Q., Fiat L., Agoulmine, N., “An Architecture for UMTS-WIMAX Interworking”, Proc of 1st IEEE Symp. on Broadband Convergence Networks, 2006, pp. $1-10$.

[11] Farahani, B.J., Ismail, M., "WiMAX/WLAN radio receiver architecture for convergence in WMANS", Proc. of 48th Midwest Symp. on Circuits and Systems, 2005, Vol. 2, pp. 1621 - 1624.

[12] 3GPP TS 25.201: "Physical layer - general description".

[13] IEEE Standard for Local and metropolitan area networks Part 16: Air Interface for Fixed Broadband Wireless Access Systems, 2004.

[14] IEEE Standard for Local and metropolitan area networks Part 16: Air Interface for Fixed and Mobile Broadband Wireless Access Systems Amendment 2: Physical and Medium Access Control Layers for Combined Fixed and Mobile Operation in Licensed Bands and Corrigendum 1, 2006 pp. 0_1 - 822.

[15] Schwartz, M. Mobile Wireless Communications, Cambridge University Press, 2005.

[16] Harju, L., Nurmi, J. A Programmable Baseband Receiver Platform for WCDMA/OFDM Mobile Terminal. Proc. of IEEE Wireless Communications and Networking Conference 2005, Vol. 1, pp. $33-38$.

[17] 3GPP TS 25.212: "Multiplexing and channel coding (FDD)".

[18] 3GPP TS 25.211: "Physical channels and mapping of transport channels onto physical channels (FDD)".

[19] http://www.ti.com

[20] http://www.lyrtech.com 